\title{
La mentoría (mentoring)
}

\section{La Relación Profesor - Alumno en la Maestría de Enfermedades Infecciosas y Zoonóticas}

La "MEIZ" es el nombre que en confianza (y casi con cariño) recibe nuestro postgrado en enfermedades infecciosas de la Escuela de Microbiología. Al momento de la publicación de este número de Portal de la Ciencia ya hemos graduado a nuestra tercera promoción de nueve Másteres en Ciencia y, si todo marcha bien, el próximo año 2019 estaremos iniciando con la cuarta promoción. Si, sé que estarán pensando que es muy poco el tiempo y la experiencia que tenemos en la MEIZ como para osar dar cátedra de nada. Estoy consciente de que nos queda mucho por caminar, errores que cometer y lecciones por aprender. Somos jóvenes (como postgrado, nosotros ya no tanto). Es por lo que les ruego que al leer estas palabras las tomen solamente como el testimonio humilde de quienes han iniciado un camino que pretende formar lo mejor posible a una nueva generación de jóvenes investigadores a nivel de postgrado para Honduras.

La MEIZ inició coyunturalmente a partir de un financiamiento que un grupo de profesoras de la UNAH y una colega honduro-canadiense, gestionaron ante el Gobierno de Canadá. Estas valientes mujeres tuvieron un sueño, que parecía quijotesco en aquel momento en que la Reforma universitaria era apenas una promesa difusa, y la convulsión socio-política del país daba lugar a todo menos a la creatividad. A pesar de los obstáculos internos y externos, el Postgrado fue creado y aprobado, pero con una mística de trabajo nueva, inspirada en la Universidad de Brock, y el resto de las Universidades canadienses. Era necesario adoptar paradigmas en los que ninguno de nosotros habíamos sido formados. Requería una transformación de la mente de los coordinadores, los profesores y los asesores, para luego intentar permear en la de los estudiantes.

Estos nuevos paradigmas estaban relacionados fundamentalmente con tres ejes: (a) la ética de la investigación, (b) la bioseguridad y (c) la rigurosidad en la investigación.

\footnotetext{
${ }^{1}$ Coordinador del Centro Experimental y de Innovación de Investigaciones Genéticas, Universidad Nacional Autónoma de Honduras. Posee una Licenciatura en Microbiología y Química Clínica, Maestría en Microbiología con énfasis en Biología Celular y Molecular y Doctorado en Genética y Biología Celular. contacto: gustavo.fontecha@unah.edu.hn
} 
Otro de esos cambios de óptica se enfocó en las relaciones entre maestros y estudiantes en la MEIZ, relaciones académicas y profesionales, pero también personales. Es de esto que me atreveré a contarles en los siguientes cinco puntos:

\section{1. "La fuerza te acompañe" ... Atención personalizada}

En mi ya lejana infancia me sentí fuertemente inspirado por la Orden "Jedi", de la trilogía "Star Wars", en la que un pequeño grupo de sabios-guerreros mantenía el equilibrio de la "República". Estos maestros Jedi eran personajes solitarios que solían tener a su cargo la formación de un joven aprendiz o "Padawan". La relación entre maestro y aprendiz era muy estrecha, era individualizada. Se basaba en el desarrollo de destrezas y conocimientos en el aprendiz, pero sobre todo en la transmisión de un "carisma", de una forma de ser, de actuar, de interpretar la vida.

Desde la primera promoción, la MEIZ se propuso que cada estudiante tuviese su propio asesor. Y que cada asesor no tuviese sino un estudiante. A pesar de las dificultades para reclutar asesores que fuesen investigadores consolidados, nos fue posible armonizar los intereses y las personalidades de los nueve estudiantes (de la primera promoción) con nueve supervisores académicos, cuatro de ellos en el extranjero. Estas relaciones personalizadas entre un estudiante de postgrado y un asesor son fundamentales para el éxito de la experiencia de aprendizaje, para que se alcance un sentido de satisfacción por las dos partes, para el desarrollo de las destrezas y habilidades que hacen a un investigador, y sobre todo para el modelaje de una potencial trayectoria profesional exitosa tanto para el estudiante como para el investigador que lo acoge.

En nuestro ambiente, esta deseable armonía se ve amenazada por el escaso o nulo entrenamiento que tenemos los profesores para asesorar tesis de postgrado en nuestra Escuela, o por que las expectativas de ambas partes no son coincidentes, o por los conflictos producidos por la natural incompatibilidad de personalidades entre estudiantes y asesores.

En un programa de posgrado es importante establecer buenas relaciones académicas, pero también es necesario considerar algún nivel de relaciones personales ("ni tanto que queme al santo ni tan poco que no lo alumbre"). Las personalidades juegan un papel importante en la tarea de supervisión de una tesis. La relación entre asesor y estudiante no solo es más estrecha que en el pregrado, sino que es de largo plazo. El éxito que tengan los estudiantes graduados en sus proyectos dependerá en gran medida de la naturaleza de las relaciones que establezca con sus maestros. 


\section{Reglas claras amistades largas}

Una de las razones más comunes por las cuales se abandonan los estudios de postgrado yace en la prolongación sin término de la investigación y / o de la escritura de la tesis. Un programa exitoso de postgrado esperaría que sus estudiantes completaran sus tareas en el tiempo preestablecido. Esto solo se logra cuando las "reglas del juego" están claras desde el inicio. Esto pasa por informar claramente a los candidatos cuál es la naturaleza del programa, qué objetivos persigue, cuál es el perfil profesional que se pretende formar en ellos y, sobre todo, lo que se espera de ellos. Luego, es necesario contar con un calendario preciso de las fechas y horarios de los cursos, así como de la fecha de entrega de los productos de la investigación que sean requeridos a lo largo del programa. Es esencial que no quede nada al azar. Recuerde que las cosas que pueden parecer obvias para los profesores pueden no ser tan obvias para los estudiantes. La supervisión del proceso debe ser estricta y debe contar con una comunicación abierta y constante entre todos los implicados. Esta claridad puede prevenir muchas frustraciones, y ayuda a garantizar que los procesos se culminen con éxito, en tiempo y forma.

\section{Como dijera Ortega y Gasset: "Yo soy yo y mis circunstancias"}

Una de las principales limitantes que encontramos al momento de crear la MEIZ fue la falta de infraestructura suficiente para la experimentación. Nuestro postgrado es en ciencias duras y ello requiere de espacio y recursos para funcionar. La construcción y equipamiento del laboratorio de investigación Teasdale-Corti fue por tanto una de las prioridades del grupo fundador. A partir de este laboratorio-semilla se ha generado recientemente el primer Centro experimental de la UNAH llamado Centro de Investigaciones Genéticas, que actualmente aporta más del $15 \%$ de toda la producción científica nacional.

Los postgrados en ciencias requieren de investigación de alta calidad, y sobre todo de un ambiente de aprendizaje que sea estimulante tanto para el estudiante graduado como para los profesores. Es tarea de los asesores, y del programa de postgrado, proveer un ambiente propicio para que sus estudiantes puedan desarrollarse plenamente como investigadores. Pero este ambiente fértil no es solamente el fruto de contar con aparatos, tecnologías de punta, y recursos materiales suficientes, sino que nace espontáneamente cuando flota en el aire un auténtico interés por la ciencia, cuando surgen interrogantes, se discute, cuando se estimula el libre pensamiento de todos. Por otro lado, un ambiente sereno, en el que existan condiciones que permitan el trabajo mano a mano, se construye con la comunicación efectiva entre maestro y 
aprendiz, el respeto interpersonal y el afecto. Como dijera Einstein: "Yo no enseño a mis alumnos, solo les proporciono las condiciones en las que puedan aprender".

\section{Aprender haciendo}

"Aprender haciendo" es un lema ya poco novedoso. Muchas Universidades en el mundo han hecho del aprendizaje basado en problemas su metodología de trabajo. Es una parte fundamental de la filosofía de la MEIZ el "arrojar a la piscina" a los que anhelan nadar. Para convertirse en científico, en investigador, es necesario pasar largas horas diseñando y realizando experimentos, analizando datos, escribiendo y comunicando resultados. Todas estas facetas de un investigador son promovidas como parte de la formación de nuestros estudiantes. Es deseable que nuestros chicos sean capaces de, a través de sus proyectos de investigación, cuestionar ideas e introducir nuevas ideas, desafiar conocimientos, explorar excentricidades, seguir corazonadas, y proponer caminos nuevos. Es esta libertad de mente la que lleva a dar saltos cualitativos en las ciencias. Evidentemente, esto puede sonar muy romántico, es cierto. Todo este romanticismo está sostenido por un andamiaje de trabajo duro, rutinario, agotador, sin el cual no es posible templar el carácter ni forjar un espíritu capaz de sobrellevar las muchas vicisitudes que nuestra realidad ofrece.

\section{Amor y rigor: "La educación es cosa del corazón" (Don Bosco)}

"Asesor" es el término que utilizamos en la MEIZ para los profesores que dirigen los trabajos de tesis; sin embargo, nuestros asesores hacen trabajos tanto de supervisión formal como de mentores. El trabajo de supervisión es complejo, requiere mucho más que buena voluntad y dedicación. Es una dirección formal, de autoridad, que vela por el cumplimiento de las metas. El rol de mentor, en cambio, incluye un tipo de relación más espiritual en el que se comunica la propia experiencia para ayudar al estudiante a alcanzar sus propias metas personales. Un mentor es transmisor informal de los conocimientos que se han ido ganando con la experiencia, con el fin de hacer crecer y madurar al aprendiz. El desarrollo personal del joven es la clave para el mentor.

Ambos roles se conjuntan en nuestros asesores. El rigor de la supervisión, y el afecto paternal (o maternal, para no dejar fuera a nadie) del mentor. Ambos son necesarios para el acompañamiento de nuestros estudiantes. Ellos requieren una guía firme, disciplina, exigencia, que les obligue a dar lo mejor de sí. Sin embargo, este rigor académico debe ir siempre lubricado por lo que los italianos llaman "amarevolezza" (amabilidad, confianza, familiaridad). Esta amabilidad hace que las relaciones se 
tornen cálidas, divertidas y despierten las energías emocionales que permiten disfrutar del trabajo de investigación, casi siempre duro y sacrificado.

Este apoyo que necesitan los estudiantes por parte de su asesor evoluciona con el tiempo. Sus necesidades cambian en la medida que estos crecen académicamente. Uno de los retos de los asesores es percibirlas: Diferenciar cuándo el estudiante necesita acompañamiento y cuándo necesita autonomía. De acuerdo a Gatfield (2005), existen cuatro tipos de relación en la supervisión de un trabajo de tesis que pueden utilizarse dependiendo de la necesidad del momento: (a) dejar hacer 0 "laissez-faire"; (b) directiva; (c) consultiva; y (d) pastoral. Existirán momentos en los que dejar hacer sea lo mejor; cuando no sean necesarios ni convenientes altos niveles de interacción por el asesor. En cambio, cuando el estudiante requiere más estructura, el asesor se convierte en un director, que guía, exige, orienta, asigna tareas y fechas de entrega. Cuando el estudiante se familiariza más con su rol y su trabajo, el rol del asesor podrá ser más como el de un consultor, que ofrece sugerencias y opciones para solventar los problemas. En las etapas más difíciles de la investigación, por ejemplo, en la recta final de la escritura de la tesis, el enfoque pastoral puede ser utilizado para brindar apoyo personal, motivación y autoconfianza. Tiene como propósito hacer que el estudiante pase de un estado mental de aprendiz a uno de profesional independiente.

En fin, con el transcurrir de los años coordinando la MEIZ, nuestro equipo de trabajo se ha ido forjando con las experiencias de la asesoría de estudiantes, algunas dolorosas, pero generalmente satisfactorias. Creemos que nuestra principal misión es inspirar. Inspirar en los jóvenes investigadores el deseo de aprender, el ímpetu por descubrir, la sensibilidad de la contemplación del conocimiento. 
